\title{
Ein soziales und demokratisches Europa? Hindernisse und Handlungsperspektiven
}

Überall in Europa schwindet die Unterstützung für den europäischen Integrationsprozess. Die Europäische Union (EU) wird mehr und mehr mit dem Abbau von sozialen und demokratischen Rechten in Verbindung gesetzt. Diese Wahrnehmung ist durchaus berechtigt. Soziale Grundrechte geraten unter das Räderwerk des marktliberalen Binnenmarktrechts, das europäische Sozialmodell wird Stück für Stück demontiert. Doch was sind die Ursachen für diese Schieflage? Wie lässt sich diese problematische Fehlentwicklung beheben? Welche Veränderungen wären für die Verwirklichung eines sozialen und demokratischen Europas notwendig?

DANIEL SEIKEL

\section{Einleitung}

Mit dem europäischen Einigungsprozess wurden immer schon große Ziele verbunden. Nach zwei verheerenden Weltkriegen wurde die Einigung Europas eng mit der Überwindung des Nationalismus und somit der dauerhaften Sicherung von Frieden auf dem europäischen Kontinent verknüpft. Von Beginn an sollte die damalige Europäische Wirtschaftsgemeinschaft (EWG) aber auch das Wachstum fördern, den Wohlstand steigern, Vollbeschäftigung ermöglichen und die sozialstaatliche Entwicklung in den Mitgliedstaaten unterstützen. Während militärische Auseinandersetzungen zwischen den Ländern der EWG schon bald keine realistische Bedrohung mehr darstellten, gewann die wirtschaftliche Dimension vor allem durch die ökonomischen Krisen seit Ende der 1960er Jahre und nicht zuletzt durch die sich beschleunigende Globalisierung mehr und mehr an Bedeutung.

Heute steht die Politik der EU in der Kritik. Beobachter bescheinigen dem Integrationsprozess eine ausgeprägte marktliberale Schieflage, die das Verhältnis von sozialer Regulierung und Marktwirtschaft immer mehr zugunsten der Letzteren verschiebt (Bieling/Deppe 2003; Gill 1998; Höpner/Schäfer 2010, 2012; Scharpf 2010a; Ziltener 1999; siehe auch Beckfield in diesem Heft).

Während sich die Mitgliedstaaten bis zum Ausbruch der Eurokrise ökonomisch angenähert haben, nehmen die innerstaatlichen Einkommensungleichheiten seit Beginn der 1980er Jahre zu (siehe Beckfield in diesem Heft). Wie Martin Heidenreich in diesem Heft darlegt, ist die Einkommensungleichheit seit Ausbruch der Krise zwar nur noch in wenigen Ländern angestiegen. Die objektiven Armutsquoten haben sich aber auf einem hohen Niveau stabilisiert und die subjektive Armut hat zum Teil dramatisch zugenommen. Betrachtet man nur die erwerbstätige Bevölkerung, lässt sich sogar ein starker Anstieg der objektiven Armut feststellen (siehe den Beitrag von Cantillon et al. in diesem Heft). Im Hinblick auf die zwischenstaatlichen Unterschiede ist der Trend zur Konvergenz mit der Krise ins Stocken geraten (Heidenreich in diesem Heft). Insbesondere zwischen den nord- und den südeuropäischen Ländern klaffen die Einkommensniveaus wieder stärker auseinander. Diese Entwicklungen sind mit den Grundprinzipien eines sozialen Europas nur schwer zu vereinbaren.

Die Politik der EU und ihre Institutionen werden vielfach als technokratisch und undemokratisch kritisiert (Bellamy 2006; Enderlein 2013; Lemb/Urban 2014; Mair 2007; Majone 2014; Oberndorfer 2012; Scharpf 2009; Streeck 2013; Urban 2013). Das aktuelle Euro-Krisenmanagement unterwirft überdies in Not geratene Mitgliedstaaten einem harten Austeritätskurs, befördert den Abbau von Sozialstaatlichkeit und Arbeitnehmerrechten und bedroht zudem die wirtschaftliche Stabilität der Eurozone (Buntenbach 2014; Busch et al. 2013; Fischer-Lescano 2014; Schulten/ Müller 2013). Das sind keine guten Nachrichten für das Projekt eines sozialen und demokratischen Europas. 
Trotz dieser äußerst problematischen Fehlentwicklungen ist der Integrationsprozess gerade auch aus einer arbeitnehmerorientierten Sicht verteidigenswert. Denn die EUIntegration bietet potenziell, d. h. im Falle des Vorhandenseins der entsprechenden politischen Mehrheiten, die historische Chance, im Zeitalter von Globalisierung und entfesselten Märkten die zwischenstaatliche kapitalistische Konkurrenz einzuhegen. Durch Zusammenarbeit auf der europäischen Ebene könnten die Voraussetzungen für transnationale Solidarität geschaffen, der gegenseitige Unterbietungswettbewerb unterbunden, Märkte reguliert und der Vorrang des Politischen und des Demokratischen vor dem Ökonomischen wiederhergestellt werden. Bislang besteht diese Option allerdings nur hypothetisch. In der Praxis trägt die europäische Integration genau zum Gegenteil bei, nicht zuletzt, indem sie mitgliedstaatliche Sozialsysteme und Produktionsregime einem direkten institutionalisierten regulativen Wettbewerb aussetzt.

Das europäische Projekt ist zweifellos eine der größten zivilisatorischen Errungenschaften des 20. Jahrhunderts. Dies gilt aber mindestens im selben Maße für den demokratischen Wohlfahrtsstaat, die Tarifautonomie oder das Streikrecht. Daher darf die europäische Integration auch nicht zum Preis von Demokratie und sozialen Rechten erkauft werden. Europa ist wichtig, Sozialstaatlichkeit, Rechtsstaatlichkeit und Demokratie sind es aber mindestens ebenso.

Zurzeit werden die sozialen Errungenschaften, die das „Europäische Sozialmodell“ auszeichnen, durch die aktuelle Entwicklungstendenz des Integrationsprozesses massiv bedroht. Die aktuell größte Bedrohung stellen die Eurokrise und die Form ihrer politischen Bearbeitung dar. Die Ursachen für die Schieflage zwischen Marktregulierung und Marktschaffung wurzeln aber auch in der institutionellen Architektur des europäischen Mehrebenensystems. Aus Sicht der Verfechter eines sozialen und demokratischen Europas gilt es, diesen Fehlentwicklungen eine Alternative entgegenzusetzen, die weder in einem unkritischen „Mehr Europa um jeden Preis“ noch in einem Rückzug auf den Nationalstaat bestehen kann. In diesem Aufsatz sollen einige Überlegungen vorgestellt werden, welche Veränderungen zur Verwirklichung eines sozialen und demokratischen Europas beitragen könnten. Dabei beschränke ich mich hauptsächlich auf institutionelle Zusammenhänge. Die Euro-Krisenpolitik und ihre Korrektur werden hier nicht vertieft behandelt. Der Beitrag von Achim Truger widmet sich diesem Thema ausführlich. Er zeigt, wie sehr der austeritätspolitische Kurs im Widerspruch zur Idee eines sozialen Europas steht. Der vorliegende Artikel konzentriert sich noch stärker auf die Behebung der Defizite der EU im „Normalzustand“. Er ist als Diskussionsvorschlag zu verstehen und soll in erster Linie als Orientierungsangebot für die politische Diskussion über die Zukunft Europas dienen.

Im Folgenden werde ich zunächst das Leitbild der Sozialen Demokratie aufgreifen und es als Blaupause für eine Neuorientierung des europäischen Integrationsprozesses zugrunde legen (Abschnitt 2). Im Anschluss sollen die wich- tigsten Hindernisse benannt werden, die einem sozialen und demokratischen Europa entgegenstehen (3). In Abschnitt 4 werden auf Grundlage dieser Analyse für drei zentrale Handlungsfelder Reformoptionen skizziert. Im letzten Abschnitt (5) werden die Vorzüge der Reformvorschläge im Vergleich zum Ist-Zustand diskutiert.

\section{Das Leitbild eines sozialen und demokratischen Europas}

Dieser Abschnitt geht der Frage nach, welchem Leitbild die europäische Integration folgen sollte. Dazu greife ich das Konzept der Sozialen Demokratie auf und schlage vor, die europäische Integration in ihren Dienst zu stellen. Dazu fasse ich im Folgenden zunächst ihre Grundelemente kurz zusammen.

Das Konzept der Sozialen Demokratie beschreibt ein demokratisch verfasstes Gemeinwesen, das „die politische Verantwortung für die soziale Sicherung der Würde des Einzelnen in seinen ökonomischen und gesellschaftlichen Lebenszusammenhängen übernimmt“" (Meyer 2002, S. 13). Damit korrespondiert das Konzept der Sozialen Demokratie mit dem Demokratieverständnis des Republikanismus (siehe hierzu Scharpf 2009). Im Gegensatz zum Leitbild der liberalen Demokratie beschränkt sich der Republikanismus nicht allein darauf, die Freiheit des Individuums gegenüber staatlichem Missbrauch zu schützen. Vielmehr zielt der Republikanismus darauf ab, die Freiheit der Bürger im und durch den Staat sicherzustellen (Meyer 2002, S. 18f.). Der Sozialen Demokratie liegt die Erkenntnis zugrunde, dass die vollständige (demokratische) Teilhabe der Bürger am wirtschaftlichen und gesellschaftlichen Leben eines Gemeinwesens durch die Garantie individueller Freiheitsrechte allein nicht gewährleistet ist. Demnach ist mit dem Status des Staatsbürgers eine grundsätzliche Gleichheit aller Bürger verbunden, die mit einer aus dem Ruder laufenden wirtschaftlichen Ungleichheit unvereinbar ist (Marshall 1992, S. 88). Denn eine über ein gewisses Maß hinausgehende ungleichmäßige Verteilung materieller Ressourcen führt dazu, dass auch politische wie wirtschaftliche Partizipations- und Gestaltungsmöglichkeiten ungleich verteilt sind (ebd., S. 57f.). Erst die Veränderung der wirtschaftlichen und sozialen Verhältnisse durch eine aktive staatliche Politik erlaubt es vielen Menschen, ihre Freiheitsrechte vollständig wahrzunehmen. Dazu müssen den politischen Gestaltungs- und Interventionsrechten des Staates gegenüber Märkten und wirtschaftlicher Macht Vorrang eingeräumt werden. Dem Konzept der Sozialen Demokratie zufolge sollen daher demokratische Grund-

1 Siehe auch Prantl (2014) 
rechte auch für den wirtschaftlich-sozialen Bereich gelten (Meyer 2002, S. 13ff.). Das Gestaltungsprimat der Demokratie gegenüber der Wirtschaft ist die Voraussetzung dafür, dass auch wirtschaftlich „Schwache“ ihre Freiheitsrechte wahrnehmen können (ebd., S. 14).

Für die Soziale Demokratie sind drei Elemente von zentraler Bedeutung: soziale Staatsbürgerrechte, Wirtschaftsdemokratie und politische Gestaltungsfähigkeit. Soziale Bürgerrechte sind ein integraler Bestandteil der Legitimationsgrundlage von modernen Demokratien. Die Gewährleistung sozialer Rechte bedeutet ein Eindringen des Status als anspruchsberechtigte Staatsbürger in ansonsten freie wirtschaftliche Vertragsbeziehungen. Marktpreise - etwa für Arbeitskraft - werden so durch das Kriterium der sozialen Gerechtigkeit korrigiert (Marshall 1992, S. 81f.). Soziale Rechte sind das wichtigste Mittel gegen materielle und politische Ungleichheit (ebd. 1992, S. 67). Verwirklicht werden sie durch leistungsfähige Sozialsysteme. Der Staat garantiert ein politisch definiertes Mindestniveau für eine Versorgung mit lebenswichtigen Gütern und Dienstleistungen und/oder ein Mindesteinkommen, das zur Deckung eines ebenfalls politisch definierten Bedarfs ausreicht (ebd. 1992, S. 71).

Das zweite institutionelle Element der Sozialen Demokratie bilden Formen der Demokratisierung der Wirtschaft, also die Ausdehnung des demokratischen Selbst- und Mitbestimmungsanspruchs auf die Ökonomie (Meyer 2002, S. 17). Demnach soll allen Teilnehmern des Wirtschaftsprozesses nicht nur die Mitgestaltung ihrer unmittelbaren Arbeitsumwelt ermöglicht, sondern ihnen auch ein Mitspracherecht bei wichtigen Allokations- und Distributionsentscheidungen eingeräumt werden. Dazu gehören Institutionen wie die Unternehmensmitbestimmung, ${ }^{2}$ die Tarifautonomie und das Streikrecht. ${ }^{3}$

Schließlich bedarf es eines gestaltungsfähigen politischen Systems, das die oben genannten Elemente verwirklichen kann. Hierfür sind drei Voraussetzungen notwendig. Erstens muss der politische Entscheidungsprozess effektiv sein. Zweitens muss das politische System in der Lage sein, die materiellen Ressourcen zu generieren, um die Umsetzung von Regulierungszielen zu finanzieren. Drittens muss die Politik rechtliche Gestaltungsfreiheit über die wirtschaftspolitische Ordnung haben (Bast/Rödl 2013, S. 7). Diese Eigenschaften lassen sich auch als Problemlösungsfähigkeit der Politik zusammenfassen.

Eine der größten Herausforderungen für die Soziale Demokratie ist die Globalisierung (Meyer 2002, S. 12). Sie untergräbt die Fähigkeit des Nationalstaates zur „demokratischen Domestizierung des Kapitalismus“ (Scharpf 1998, S. 151). Dem internationalen Wettbewerb um mobile Produktionsfaktoren ausgesetzt, verliert der Nationalstaat seine Fähigkeit, im nationalen Rahmen die Voraussetzungen für Soziale Demokratie zu erhalten. Stehen Volkswirtschaften in einem offenen, unregulierten Wettbewerb, treten Probleme auf, die dazu führen können, dass Nationalstaaten über Zeit weniger sozial werden (Rückbau von Wohlfahrtsstaaten, Verschlechterung von Arbeitsbedingungen). Damit sind vor allem gegenseitige Unterbietungswettbewerbe bei Steuern (siehe den Beitrag von Rixen in diesem Heft), Sozialleistungen, Löhnen, Arbeitnehmerrechten und Umweltstandards gemeint.

Der europäische Integrationsprozess bietet zumindest hypothetisch, wenn der politische Wille bestünde, die Möglichkeit, diesen Problemen Herr zu werden: Durch internationale Kooperation auf der europäischen Ebene kann der wechselseitige Unterbietungswettbewerb unterbunden werden, indem regulative „Sperrklinken“ eingezogen werden, die verhindern, dass die Mitgliedstaaten ihre Wettbewerbsfähigkeit auf Kosten von Sozial-, Arbeits- und Umweltstandards steigern. Somit bietet die europäische Integration die Chance, die Rahmenbedingungen für die Verwirklichung von Sozialer Demokratie in einer globalisierten Welt zu schaffen und die verloren gegangenen politischen Gestaltungsspielräume zurückzuerobern. Die Betonung dieses Potenzials soll aber nicht verdecken, dass die aktuelle Entwicklungstendenz des europäischen Integrationsprozesses in genau die gegenteilige Richtung weist - eine systematische Unterminierung der zentralen Säulen der Sozialen Demokratie (für ausführliche Analysen der Ursachen siehe Höpner 2013; Höpner/Schäfer 2010, 2012; Scharpf 1999, 2010a, 2010b). Einige Beobachter gehen sogar davon aus, dass das soziale und demokratische Potenzial der EU grundsätzlich begrenzt ist und schätzen daher die Möglichkeit, einen Kurswechsel der europäischen Politik herbeizuführen, zumindest mittelfristig als gering ein (Höpner 2013; Scharpf 2010a). Für diesen Aufsatz stellt sich die Frage, was - ungeachtet der momentanen Realisierungschancen - verändert werden müsste, um bestehende Hindernisse für ein soziales und demokratisches Europa zu beseitigen.

\section{Institutionelle Hindernisse für ein soziales und demokratisches Europa}

In diesem Abschnitt sollen diejenigen institutionellen Hürden dargestellt werden, die der Verwirklichung eines sozialen und demokratischen Europas entgegenstehen. Dabei konzentriere ich mich vornehmlich auf Hindernisse, die in der Architektur des europäischen Mehrebenensystems angelegt sind.

Das erste Hindernis ist die eingeschränkte Problemlösungsfähigkeit der europäischen Politik (positive Inte-

2 In der Vergangenheit hat es sich als schwierig erwiesen, für eine Unternehmensmitbestimmung nach deutschem Vorbild Unterstützung auf europäischer Ebene zu finden. Angesichts dessen müsste gewährleistet sein, dass zwar gemeinsame europäische Lösungen ermöglicht werden, diese aber nationale Errungenschaften nicht gefährden.

3 Der Aspekt der Wirtschaftsdemokratie wird in diesem Aufsatz nicht weiter vertieft (siehe hierzu Urban 2011). 
gration). Legislativakte der Union erfordern die Zustimmung einer qualifizierten Mehrheit im Rat, je nach Politikbereich sogar Einstimmigkeit, sowie die Zustimmung einer Mehrheit im Europäischen Parlament (EP). Vertragsänderungen müssen von nationalen Parlamenten und zum Teil sogar in Referenden ratifiziert werden. Die institutionellen Hürden europäischer Gesetzgebung sind demnach außergewöhnlich hoch, die Anzahl an Veto-Punkten groß. Politiken, die der Verwirklichung eines sozialen Europas dienen könnten, lassen sich jedoch nur im Rahmen dieses politischen Entscheidungsmodus verwirklichen (Meyer 2002, S. 156). Die Schwierigkeiten der Mehrheitsbildung werden durch die politökonomische und institutionelle Heterogenität der 28 Mitgliedstaaten drastisch erhöht (Höpner 2013; Höpner/Schäfer 2008). Besonders wenn Kernelemente der nationalen Produktionsregime betroffen sind, ist eine Einigung schwierig. Dies gilt insbesondere für Fragen, die Sozial-, Steuer-, Finanz- und Tarifvertragssysteme betreffen, aber auch für die Unternehmenskontrolle einschließlich der Mitbestimmung. In einigen dieser für die Soziale Demokratie so zentralen Politikfeldern hat die EU sogar überhaupt keine direkte Regelungskompetenz. Die politische Gestaltungsfähigkeit der europäischen Politik ist also gerade in den Kernbereichen der Sozialen Demokratie eingeschränkt (vgl. hierzu Höpner 2013, S. 75f.; Meyer 2002, S. 156; Scharpf 2006, 2008a, S. 754ff.).

Das zweite Grundproblem ist die aus dem Ufer laufende Rechtsprechung des Europäischen Gerichtshofes (EuGH). Während der politische Entscheidungsmodus auf der europäischen Ebene nur eingeschränkt gestaltungsfähig ist, ist der rechtliche Integrationsmodus (negative Integration) äußerst effektiv (Höpner 2014). Die Grundlage dieses Modus, das europäische Vertragsrecht, ist hinsichtlich seiner ordnungspolitischen Ausrichtung keineswegs neutral; es zeichnet sich durch eine Dominanz vor allem individueller wirtschaftlicher Freiheitsrechte aus. Europäisches Recht ist dabei nationalem Recht, sogar dem nationalen Verfassungsrecht, übergeordnet. Die vier sogenannten Grundfreiheiten, die den freien grenzüberschreitenden Verkehr von Gütern, Personen, Dienstleistungen und Kapital garantieren, verstärken den Standortwettbewerb zwischen den nationalen Regulierungssystemen. Sie sind im Verlaufe des Integrationsprozesses zu „höchst wirksame[n] Instrumente[n] wirtschaftlicher Liberalisierung" geworden (Höpner 2013, S. 78). Für diese "Radikalisierung der Binnenmarktintegration“ (Scharpf 2008b) ist vor allem die Rechtsprechung des EuGH verantwortlich (siehe im Einzelnen Höpner 2008). Theoretisch können alle nationalstaatlichen Praktiken, Institutionen und Regulierungen, die den grenzüberschreitenden Handel auch nur potenziell unattraktiver machen, vom EuGH aufgehoben werden. Hiervon sind Formen der sozialen Marktregulierung besonders betroffen, die naturgemäß individuelle ökonomische Freiheitsrechte einschränken. Der daraus resultierende marktliberale Drall unterwandert die für die Soziale Demokratie so zentralen kollektiven und sozialen Rechte (Scharpf 2008a, 2009, 2010b; Seikel/Absenger 2015).
Die marktliberale Stoßrichtung der Integration durch Recht hat dabei nicht zu unterschätzende Konsequenzen für die Kräfteverhältnisse zwischen gesellschaftlichen Gruppen. Akteure, die ein Interesse an Liberalisierung haben, können dieses „über die Bande“ europäischen Rechts durchsetzen und damit zuvor in der nationalen Arena nicht zu überwindenden politischen Widerstand rechtlich umgehen. Akteuren, die ein Interesse an sozialer Regulierung haben - wie z. B. Gewerkschaften -, steht diese Option nicht zur Verfügung (vgl. Scharpf 2010a, S. 221f.).

Die gleichen Hürden, die den positiven Integrationsmodus blockieren, erschweren auch eine politische Korrektur der EuGH-Rechtsprechung. Fällt der EuGH ein Urteil auf Grundlage des europäischen Primärrechts, bedarf eine Korrektur der Einstimmigkeit aller Regierungen im Rat.

Weil die Einigung auf marktkorrigierende Politiken schwierig ist und richterrechtliche Integration auf Grundlage der Binnenmarktfreiheiten so effektiv, aber nahezu immer marktschaffend, verschiebt sich das Verhältnis zwischen Staat und Markt systematisch zugunsten des Marktes (Scharpf 2008a, S. 49).

Die hier geschilderten, vornehmlich institutionellen, Hürden behindern die Entstehung eines sozialen Europas. So ist die Schwäche der positiven Integration eine Ursache z. B. dafür, dass in der EU weder der Steuerwettbewerb unterbunden noch Finanzmärkte effektiv reguliert werden (siehe die Beiträge von Rixen und Nölke in diesem Heft). Die Wucht der negativen Integration hingegen erzeugt einen starken Liberalisierungsimpuls. Sie verstärkt nicht nur zwischenstaatliche Unterbietungswettbewerbe - z. B. bei Steuern -, sondern sie begrenzt zugleich durch ihre marktschaffendeStoßrichtung rechtlich sozial- und wirtschaftspolitische Handlungsspielräume in den Mitgliedstaaten. Die auf mitgliedstaatlicher Ebene verloren gegangene politische Gestaltungsfähigkeit wird aber aufgrund der Blockadeanfälligkeit des positiven Integrationsmodus nicht durch eine zusätzliche Regulierungskapazität auf der europäischen Ebene ausgeglichen. Die Folge ist eine „doppelte Asymmetrie“ von Liberalisierung und sozialer Regulierung auf europäischer und nationaler Ebene (Scharpf 2010a).

\section{Handlungsfelder für ein soziales und demokratisches Europa}

\subsection{Institutionelle Reformen: eine „offene“ Verfassung für Europa}

Dieser Abschnitt beschäftigt sich mit Lösungsvorschlägen, die an den hier herausgearbeiteten Problemen einer geringen politischen Gestaltungsfähigkeit und einer aus dem Ruder laufenden Integration durch Recht ansetzen. Wie Bast und Rödl (2013, S. 9) feststellen, besteht in Europa die 
derzeit vordringlichste Aufgabe darin, die Gestaltungshoheit demokratischer Gesetzgebung gegenüber dem Binnenmarktrecht wiederherzustellen. Sie formulieren dazu zwei Schlüsselfragen: Erstens, wie kann die „Ausübung mitgliedstaatlicher Kompetenzen vor dysfunktionalen Begrenzungen durch das Binnenmarktrecht abgeschirmt werden [...], ohne zugleich wichtige Errungenschaften der wirtschaftlichen Integration zu gefährden?" (ebd.). Zweitens, wie muss die institutionelle Architektur der EU verändert werden, um politische Gestaltungsmöglichkeiten zu gewinnen, ohne die EU „dabei legitimatorisch und institutionell zu überfordern“? (Bast/Rödl 2013, S. 9).

Fritz Scharpf (2015) hat dazu unlängst einen weitreichenden Vorschlag vorgelegt. Scharpf schlägt einen konstitutionellen Neustart vor, der die politische Gestaltungsfähigkeit auf europäischer und nationaler Ebene erweitern sowie eine Korrektur der Integration durch Recht ermöglichen soll. Das Ziel ist eine Neuordnung der institutionellen Architektur der EU, die, anders als der derzeitige Zustand, „offen“ gegenüber alternativen ordnungspolitischen Leitbildern ist - so auch gegenüber dem der Sozialen Demokratie. Der Vorschlag beruht dabei auf der Grundidee, die multiplen Veto-Punkte des europäischen Gesetzgebungsprozesses gegen Opt-out-Möglichkeiten auszutauschen, also Mitgliedstaaten zu erlauben, gegebenenfalls an einzelnen Gesetzgebungsmaßnahmen nicht teilzunehmen.

Der Vorschlag von Scharpf enthält vier Grundelemente. Das erste Element ist die De-Konstitutionalisierung des Binnenmarktrechts. Demnach würde eine europäische Verfassung auf die Bestandteile reduziert werden, die typischerweise in Verfassungen enthalten sind: Bestimmungen zu Institutionen, Verfahren und Kompetenzen sowie soziale und staatsbürgerliche Grundrechte. Das Binnenmarktrecht wäre ausdrücklich nicht Bestandteil des Verfassungsrechts, sondern bliebe im „einfachen“ Recht des acquis in Kraft, verlöre also seinen derzeitigen quasi-verfassungsrechtlichen Rang (Scharpf 2015, S. 401). Die De-Konstitutionalisierung des Binnenmarktrechtes hätte zur Folge, dass das Primat wirtschaftlicher Freiheitsrechte über soziale Kollektivrechte aufgehoben würde.

Zweitens sieht der Vorschlag vor, das monopolisierte Initiativrecht der Kommission abzuschaffen. Stattdessen würde es auch qualifizierten Minderheiten im EP und im Rat ermöglicht, Gesetze vorzuschlagen (Scharpf 2015, S. 400). Dies würde verhindern, dass die Kommission Maßnahmen, die etwa den marktliberalen Präferenzen der Generaldirektionen Binnenmarkt, Wettbewerb oder Handel nicht entsprechen, verhindern oder verwässern kann (Scharpf 2014, S. 19). Außerdem würde das EP deutlich aufgewertet und damit ein wichtiger Beitrag zur Demokratisierung der EU geleistet.

Der dritte Bestandteil des Vorschlages ist eine Senkung der vergleichsweise hohen Mehrheitserfordernisse für die einfache Gesetzgebung. Demnach würde für die Annahme von Gesetzesvorschläge eine einfache Mehrheit jeweils im Rat und im EP ausreichen (Scharpf 2015, S. 400).
Dieser Vereinfachung des Entscheidungsprozesses stünde viertens die Möglichkeit von politisch kontrollierten Optouts von Mitgliedstaaten (bei einfacher Gesetzgebung) gegenüber. ${ }^{4}$ Dies ist im Sinne eines Minderheitenschutzes geboten: Einzelne oder kleine Gruppen von Mitgliedstaaten mit besonderen nationalen Eigenheiten - z. B. einer besonders weitreichenden Unternehmensmitbestimmung - die nicht mehrheitsfähig sind, können so davor geschützt werden, ständig durch einfache Mehrheiten der übrigen Staaten (z. B. beim Übernahmerecht oder Gesellschaftsrecht) überstimmt zu werden. Das Opting-out müsste dabei aber politisch kontrolliert werden. Opt-outs könnten demnach von einer absoluten Mehrheit im Parlament und einer qualifizierten Mehrheit im Rat abgelehnt werden. Dies kann etwa dann sinnvoll sein, wenn eine einheitliche Anwendung von Gesetzen notwendig erscheint, z. B. dann, wenn Nicht-Anwender einen Wettbewerbsvorteil erhielten und/oder der Nutzen der Anwender durch nicht-einheitliche Anwendung reduziert würde. In diesen Fällen müssten die Mitgliedstaaten zwischen einer vergleichsweise unproblematischen Annahme möglichst weitreichender Lösungen durch eine kleinere Gruppe von Ländern einerseits und einer langwierigen und blockadeanfälligen Kompromisssuche mit allen Mitgliedstaaten andererseits abwägen, bei der am Ende womöglich nur der kleinste gemeinsame Nenner zustande käme (Scharpf 2015, S. 402f.). Der Vorschlag sieht zudem vor, dass Mitgliedstaaten Gesetze erlassen können, die nicht im Einklang mit dem gegenwärtigen acquis sind, da darin zurzeit mehr einheitliche Regeln enthalten sind als nach der hier skizzierten Reform beschlossen würden. Solche Abweichungen müssten zuvor der Kommission gemeldet werden und könnten ebenfalls von qualifizierten Mehrheiten im EP und im Rat abgelehnt werden (Scharpf 2015, S. 403f.). ${ }^{5}$

Diese Maßnahmen adressieren die zuvor identifizierten Probleme einer geringen politischen Problemlösungsfähigkeit und einer unkontrollierten Integration durch Recht. Erstens würde die Rechtsentwicklung politisch kontrollierund korrigierbar. Die politischen Gestaltungsspielräume der Mitgliedstaaten könnten so von solchen europarechtlichen Beschränkungen befreit werden, die nicht länger den gemeinsamen Interessen politischer Mehrheiten entsprechen (Scharpf 2015, S. 404). Die europäische Politik würde von den rechtlichen Zwängen einer verselbstständigten Integration durch Recht befreit werden. Dies würde die Band-

\footnotetext{
4 Vergleiche hierzu auch das Konzept von Piris (2012) zu einem Europa der zwei Geschwindigkeiten.

5 Opt-outs ähneln zwar dem schon jetzt möglichen Verfahren zur „verstärkten Zusammenarbeit", haben aber den Vorzug, dass Beschlüsse mit Opt-out-Option Bestandteil des aqcuis werden, siehe hierzu Piris (2012, S. 62). Außerdem unterliegen Maßnahmen im Rahmen der "verstärk ten Zusammenarbeit" dem europäischen Vertragsrecht. Scharpfs Vorschlag zielt hingegen auf eine Aufweichung der aus dem europäischen Recht erwachsenden Beschränkungen für den politischen Entscheidungsprozess.
} 
breite politischer Alternativen insgesamt deutlich vergrößern. Zweitens würden die institutionellen Beschränkungen des Multi-Veto-Systems der Gemeinschaftsmethode beseitigt. Dies würde nicht nur die politische Gestaltungsfähigkeit erhöhen und ebenfalls die Korrigierbarkeit der Rechtsentwicklung erleichtern, sondern auch zu einer stärkeren öffentlichen Aufmerksamkeit für und zu einer Politisierung von europäischen Entscheidungen auf europäischer wie auf nationaler Ebene beitragen (Scharpf 2015, S. 404). Damit würde auch eine aktivere europapolitische Rolle nationaler Parlamente einhergehen und somit ein Beitrag zur Verbesserung der demokratischen Qualität in den Mitgliedstaaten geleistet (vgl. hierzu Urban 2013, S. 143f.).

Durch diese Maßnahmen wäre freilich noch lange nicht die Verwirklichung eines sozialen Europas garantiert. Dies kann nur das Ergebnis von erst noch auszufechtenden politischen Auseinandersetzungen sein. Aber zumindest würden der Sozialen Demokratie entgegenstehende institutionelle Hindernisse beseitigt und somit überhaupt erst die Voraussetzungen dafür geschaffen, dass politische Mehrheiten in Europa in demokratisch legitimierten Verfahren alternative sozial- und wirtschaftspolitische Konzeptionen umsetzen könnten.

\subsection{Mindestbedingungen für ein soziales Europa}

Für die Verwirklichung eines sozialen und demokratischen Europas wäre es notwendig, die europäische Integration stärker in den Dienst der Förderung der wohlfahrtsstaatlichen Entwicklung in den Mitgliedstaaten zu stellen. Gerade in der gegenwärtigen Krise kommt diesem Aspekt eine größere Bedeutung zu denn je. Die soziale Ungleichheit in den Mitgliedstaaten der EU ist bis zum Ausbruch der Krise angestiegen (siehe die Beiträge von Beckfield und Heidenreich in diesem Heft). Wie oben aufgeführt, ist eine hohe materielle Ungleichheit unvereinbar mit den Grundsätzen der Sozialen Demokratie. Statt wie derzeit auf den Abbau von sozialen Leistungen und die Senkung von Löhnen zu setzen, müssten daher soziale Mindeststandards vereinbart werden, die eine ausreichende Absicherung gegenüber sozialen Risiken bieten, aber auch einen angemessenen Lebensstandard gewährleisten. Dabei ginge es ausdrücklich nicht um die Einführung von Sozialversicherungssystemen auf der europäischen Ebene, sondern um regulative Vorgaben, die die Mitgliedstaaten umsetzen würden (siehe den Beitrag von Cantillon et al. in diesem Heft).

In Anlehnung an das „Korridormodell“ von Busch (2005, S. 43ff.) könnten beispielsweise Mindest-Lohnersatzraten für Sozialleistungen wie Arbeitslosengeld und Rente eingeführt werden. Ebenso wären Vorgaben für die Mindestsicherung denkbar. Um die Sozialsysteme der Länder nicht finanziell zu überfordern, könnten die Mitgliedstaaten entsprechend ihrer wirtschaftlichen Leistungsfähigkeit in Gruppen mit unterschiedlich hohen Lohnersatzraten eingeteilt werden, die langfristig an ein höheres Niveau angepasst werden sollten.
Die Krise in den südeuropäischen Ländern hat verdeutlicht, dass in der EU selbst die Versorgung mit grundlegenden Dienstleistungen nicht gewährleistet ist. Vor diesem Hintergrund erscheint es ebenfalls sinnvoll, Mindestanforderungen für die öffentliche Daseinsvorsorge festzulegen. Dabei ginge es um die Absicherung basaler Bedürfnisse wie etwa eine garantierte Grundversorgung mit Strom und Gas oder eine flächendeckende Gesundheitsversorgung.

Ein weiterer Baustein könnte eine europaweit koordinierte Mindestlohnpolitik sein (Schulten 2015; siehe auch den Beitrag von Schulten in diesem Heft). Dies wäre ein wirksames Mittel gegen die steigende soziale Ungleichheit und wachsende Armut trotz Arbeit. Angesichts der immensen Lohnunterschiede zwischen den Mitgliedstaaten kann es sich dabei allerdings nicht um einen europaweit einheitlichen Mindestlohnbetrag handeln, sondern um eine Mindestlohnnorm, die dann in den Mitgliedsländern umgesetzt wird (Schulten 2015, S. 173), wo dies von Gewerkschaften gewünscht wird. Das Mindestlohnniveau könnte beispielsweise auf $60 \%$ des jeweiligen Medianlohns eines Landes festgelegt werden.

\subsection{Stärkung der fiskalischen Handlungsfähigkeit}

Eine wichtige Voraussetzung für eine hohe Problemlösungsfähigkeit von politischen Systemen besteht in der Fähigkeit politischer Gemeinwesen, auch die materiellen Ressourcen $\mathrm{zu}$ generieren, die für die Umsetzung anspruchsvoller Regulierungsziele benötigt werden. Mit anderen Worten, politische Gestaltungsfähigkeit setzt fiskalische Handlungsfähigkeit voraus. Hierbei sind vor allem zwei eng miteinander verzahnte Probleme zu lösen, die die Einnahmeseite staatlicher Haushalte beeinträchtigen: Die Regulierung von Finanzmärkten und die Eindämmung des Steuerwettbewerbs.

Wie in den Beiträgen von Nölke und Rixen in diesem Heft deutlich wird, ist die Einhegung von Finanzmärkten und des Steuerwettbewerbs eine wichtige Voraussetzung für ein soziales und demokratisches Europa. Beide Beiträge verweisen auf den engen Zusammenhang zwischen Finanzialisierung und Steuerwettbewerb einerseits und sozialer Ungleichheit andererseits. Überdies entziehen Finanzialisierung, Steuervermeidung und Steuerwettbewerb den Nationalstaaten in erheblichem Umfang dringend benötigte Ressourcen (siehe hierzu Unger 2013). Die Liberalisierung von Kapitalströmen und Finanzmärkten hat dazu geführt, dass sich die Mobilität des Faktors Kapital stark erhöht hat. Finanzmarktakteure, Unternehmen und Vermögende können die Unterschiede zwischen nationalen Regulierungsmodellen ausnutzen, um eine „regulative Arbitrage“ zu erzielen. Nationalstaaten geraten dadurch in einen Standortwettbewerb um Investitionen und Steuereinnahmen. Aufgrund der vergleichsweise weit fortgeschrittenen Marktliberalisierung ist dieser Standortwettbewerb in der EU besonders intensiv. Der gegenseitige Unterbietungswettlauf zwischen den Mitgliedstaaten wird so begünstigt. Legale 
wie illegale Möglichkeiten der Steuervermeidung sowie eine zunehmend kapitalfreundliche Finanzmarktregulierung und Besteuerung verringern dabei nicht nur die Steuereinnahmen, sondern führen auch generell zu einem Verlust politisch-demokratischer Kontrolle über das Finanzmarktgeschäft.

In seinem Beitrag fordert Nölke eine stärkere staatliche Kontrolle des grenzüberschreitenden Kapitalverkehrs. Dies würde nicht nur die Krisenanfälligkeit des Finanzmarktgeschäfts verringern, sondern auch die Besteuerung von Kapitaleinkünften und Unternehmensgewinnen erleichtern. Wie Nölke feststellt, wäre diese Maßnahme allerdings nicht mit der primärrechtlich verankerten Kapitalverkehrsfreiheit vereinbar. Außerdem befürwortet Nölke die Einführung einer Finanztransaktionssteuer. Sie würde ebenfalls zur Entschleunigung des Finanzmarktgeschäfts beitragen und zusätzliche öffentliche Einnahmen generieren. Der Finanzsektor würde auf diese Weise stärker an der Finanzierung öffentlicher Aufgaben beteiligt. Daneben ist eine effektivere Regulierung von Banken, Schattenbanken und Finanzinstrumenten notwendig.

Wie Rixen in seinem Beitrag zeigt, hat es in der EU zwar Fortschritte bei der Bekämpfung von Steuerhinterziehung gegeben. Erhebliche Defizite bestehen aber weiterhin bei der Einhegung des Steuerwettbewerbs. Rixen schlägt hierzu eine Teil-Harmonisierung der Steuerpolitik vor und plädiert für die Besteuerung von Unternehmensgewinnen in Form einer gemeinschaftlich konsolidierten Körperschaftsteuerbemessungsgrundlage in Kombination mit Mindeststeuersätzen.

\section{Fazit}

In diesem Beitrag habe ich vorgeschlagen, die europäische Integration in den Dienst der Sozialen Demokratie zu stellen. Die Grundidee dabei ist, regulative „Sperrklinken“ gegen den gegenseitigen Unterbietungswettbewerb einzuziehen und den Integrationsprozess so auszurichten, dass er im Sinne einer komplementären institutionellen Regulierungsebene die wirtschaftliche und wohlfahrtsstaatliche Entwicklung in den Mitgliedstaaten absichert und fördert. Dazu habe ich zunächst dargestellt, wie die derzeitige Architektur des europäischen Mehrebenensystems die Verwirklichung von Sozialer Demokratie in Europa systematisch verhindert. Ausgehend von dieser Analyse habe ich drei Handlungsfelder identifiziert, die aus meiner Sicht für ein soziales und demokratisches Europas von entscheidender Bedeutung sind: eine „offene“ europäische Verfassung, Mindestbedingungen für ein soziales Europa und die Stärkung der fiskalischen Handlungsfähigkeit der Politik.

Es sei darauf hingewiesen, dass sich keiner der hier gemachten Vorschläge leicht umsetzen lassen wird, weil zunächst die in Abschnitt 3 beschriebenen Hürden überwun- den werden müssten. Auch sind Zweifel daran angebracht, ob es derzeit in Europa überhaupt die politischen Mehrheiten gibt, die derartige Reformen in die Tat umsetzen würden. Das gilt auch und im besonderen Maße für den Vorschlag eines konstitutionellen Neustarts der EU (Scharpf 2015, S. 397). Nach Scharpfs Einschätzung bräuchte es dazu sogar eine dramatisch zugespitzte Krisensituation, die die politischen Akteure dazu bewegen würde, ihre Blockadepositionen aufzugeben und somit einen umfassenden institutionellen Wandel jenseits von inkrementellen, pfadabhängigen Anpassungen zu ermöglichen.

Die hier umrissene institutionelle Reform würde gleichwohl die Erfolgschancen der übrigen Vorschläge erheblich erhöhen, auch wenn sie für einige der vorgeschlagenen Maßnahmen keine unbedingt zwingende Voraussetzung ist. So ist zumindest vorstellbar, dass auch unter den gegenwärtigen institutionellen Bedingungen eine Einigung auf proportionale Mindestbedingungen im Sozialbereich möglich wäre. Opt-outs könnten aber dennoch Fortschritte in diesem Bereich erleichtern. Bestes Beispiel hierfür ist das Thema einer europäischen Mindestlohnnorm. Eine gemeinsame Mindestlohnpolitik wurde bislang im Europäischen Gewerkschaftsbund (EGB) vor allem von einigen derjenigen Länder abgelehnt, die eine hohe Tarifbindung sowie ein hohes Lohnniveau aufweisen und befürchten, dass europäische Vorgaben zu einem Absinken ihrer nationalen Standards führen könnte (siehe hierzu Seeliger 2015). ${ }^{6}$ Die Möglichkeit eines Opt-outs könnte diese Blockade auflösen, ohne dass dadurch gemeinsame Standards unterwandert würden, da es sich bei den Nicht-Teilnehmern um Länder handeln würde, die ohnehin hohe Lohnniveaus aufweisen.

Eine Einigung auf strenge Finanzmarktregulierungen sowie auf eine Teil-Harmonisierung der Körperschaftssteuer ist hingegen angesichts grundsätzlicher nationaler Interessenunterschiede derzeit äußerst unwahrscheinlich. In diesen Politikfeldern würde ein Vorankommen durch Optouts überhaupt erst ermöglicht.

Zweifellos wären in den genannten Politikfeldern einheitliche Regelungen am effektivsten, um gegenseitige Unterbietungswettbewerbe wirksam einzuhegen. Fortschritte einer „Avantgarde“-Gruppe von Ländern in den für ein soziales Europa zentralen Bereichen erscheinen jedoch immer noch besser als gar keine Verbesserungen. Hierbei ist wichtig zu betonen, dass der hier unterbreitete Reformvorschlag eine politische Kontrolle von Opt-outs vorsieht. Diese sollen von einer qualifizierten Mehrheit im Rat abgelehnt werden können. Dies bedeutet, wenn eine einheitliche Regelanwendung erforderlich erscheint, fallen die Mit-

\footnotetext{
6 Auf dem EGB-Kongress 2015 haben sich die Gewerkschaften auf eine Kompromissformel verständigt: Der EGB unterstützt die Einführung von Mindestlöhnen, wenn dies von nationalen Gewerkschaften gefordert wird. Dies ist nicht als eine Forderung nach einem einheitlichen europäischen Mindestlohn zu verstehen. Der gefundene Kompromiss zeigt das Potenzial von Opt-out-Lösungen auf.
} 
gliedstaaten im Prinzip auf die Verfahrenslogik der (klassischen) Gemeinschaftsmethode zurück. Die Reform würde also in keinem Fall zu einer Verschlechterung im Vergleich zum ,integrativen Ist-Zustand“ führen, wohl aber neue, derzeitig versperrte Handlungsoptionen eröffnen. In den Bereichen, in denen gegenwärtig Einstimmigkeit erforderlich ist wie z. B. in der Steuerpolitik, würde die Konsensfindung sogar deutlich erleichtert, da Mitgliedstaaten ihr jeweiliges Veto-Recht verlieren würden. Zudem würde auch das EP eine pro-aktivere Agenda-Setting-Rolle einnehmen und zentrale Politikvorhaben initiieren können. Überdies wären zurzeit bestehende europarechtliche Beschränkungen (z. B. Kapitalverkehrsfreiheit) politisch umgehbar.

Der vorliegende Beitrag ist in erster Linie als Diskussionsvorschlag gedacht. Er soll Handlungsperspektiven identifizieren und ausgewählte Lösungskonzepte skizzieren. Er beantwortet nicht die Frage, wie der Weg zu einem sozialeren und demokratischeren Europa aussehen könnte, also, wie sich die Vorschläge in die Tat umsetzen lassen. Der Zweck des Beitrages besteht demnach auch darin, für den Zeitpunkt, in dem sich in Europa ein Gelegenheitsfenster für grundlegende Reformen öffnet, konkrete Orientierungshilfen aufzuzeigen - nicht zuletzt, um auf die Zeit nach der nächsten Krise besser vorbereitet zu sein (vgl. Scharpf 2015, S. 397).

Die hier skizzierten mittel- bis langfristig angelegten Reformperspektiven sollen auch nicht den Blick darauf verstellen, dass die aktuell vordringlichste Aufgabe der Schutz nationaler Sozialsysteme und Tarifordnungen vor illegitimen Übergriffen aus Brüssel und Luxemburg ist. Wenn auch im Rahmen dieses Beitrages nicht näher behandelt, ist die derzeitig größte Bedrohung für ein soziales und demokratisches Europa zweifellos die gegenwärtige Euro-Krisenpolitik. Hier ist ein grundlegender Kurswechsel dringend geboten (siehe hierzu den Beitrag von Truger in diesem Heft). Ein wichtiger Schlüssel hierfür liegt in Deutschland: Als größter Kreditgeber übt Deutschland einen nicht zu unterschätzenden Einfluss auf das europäische Krisenmanagement aus (Bulmer 2014, S. 1249f.). Eine Kurskorrektur der deutschen Europapolitik wäre daher eine entscheidende Voraussetzung für eine Neuorientierung der europäischen Krisenpolitik.

\section{LITERATUR}

Bast, J./Rödl, F. (2013): Wohlfahrtsstaatlichkeit und "soziales Europa“ im Kontext der Euro-Krise, in: Europarecht Beiheft (1) 2013, S. 5-12

Bellamy, R. (2006): Still in deficit: in: European Law Journal 12 (6), S. 725-742 Bieling, H.-J./Deppe, F. (2003): Die neue europäische Ökonomie und dieTransformation von Staatlichkeit, in: Jachtenfuchs, M./Kohler-Koch, B. (Hrsg.): Europäische Integration, Opladen, S. 513-539

Bulmer, S. (2014): Germany and the eurozone crisis: between hegemony and domestic politics, in: West European Politics 37 (6), S. 1244-1263

Buntenbach, A. (2014): Wer hat Recht(e) in Europa?, in: Supplement der Zeitschrift Sozialismus 4/2014, S. 3-11

Busch, K. (2005): Die Perspektiven des Europäischen Sozialmodells, Düsseldorf

Busch, K./Hermann, C./Hinrichs, K./Schulten, T. (2013): Euro crisis, austerity policy and the European Social Model. How crisis policies in southern Europe threaten the EU's social dimension: Friedrich-Ebert-Stiftung, International Policy Analysis, February

Enderlein, H. (2013): Das erste Opfer der Krise ist die Demokratie: Wirtschaftspolitik und ihre Legitimation in der Finanzmarktkrise 2008-2013, in: Politische Vierteljahresschrift 54 (4), S. 714-739

Fischer-Lescano, A. (2014): Austeritätspolitik und Menschenrechte - Rechtspflichten der Unionsorgane beim Abschluss von Memoranda of Understanding Rechtsgutachten im Auftrag der Kammer für Arbeiter/innen und Angestellte fürWien

Gill, S. (1998): European Governance and new constitutionalism, in: New Political Economy 3 (1), S. 5-26
Höpner, M. (2008): Usurpation statt Delegation. Wie der EuGH die Binnenmarktintegration radikalisiert und warum er politischer Kontrolle bedarf, MPIfG Discussion Paper 08/12, Köln

Höpner, M. (2013): Soziale Demokratie?, in: Europarecht Beiheft 1/2013, S. $69-89$

Höpner, M. (2014): Wie der Europäische Gerichtshof und die Kommission Liberalisierung durchsetzen: Befunde aus der MPIfG-Forschungsgruppe zur Politischen Ökonomie der europäischen Integration, MPIfG Discussion Paper 14/8, Köln

Höpner, M./Schäfer, A. (2008): Eine neue Phase der europäischen Integration. Legitimationsdefizite europäischer Liberalisierungspolitik, in: Höpner, M./ Schäfer, A. (Hrsg.): Die Politische Ökonomie der europäische Integration, Frankfurt a. M., S. 129-156

Höpner, M./Schäfer, A. (2010): A new phase of European integration, in: West European Politics 33 (2), S. 344-368

Höpner, M./Schäfer, A. (2012): Embeddedness and regional integration, in: International Organization 66 (3), S. 429-455

Lemb, W./Urban, H.-J. (2014): Ist die Demokratie noch zu retten?, in: Supplement der Zeitschrift Sozialismus 4/2012, S. 42-54

Mair, P. (2007): Political opposition and the European Union, in: Government and Opposition 42 (1), S. 1-17

Majone, G. (2014): From regulatory state to a democratic default, in: Journal of Common Market Studies 52 (6), S. 1216-1223

Marshall, T. H. (1992): Bürgerrechte und soziale Klassen, Frankfurt a. M.

Meyer, T. (2002): Soziale Demokratie und Globalisierung. Eine europäische Perspektive, Bonn

Oberndorfer, L. (2012): Der Fiskalpakt - Umgehung der "europäischen Verfassung” und Durchbrechung demokratischer Verfahren?, in: juridikum 2/2012,

S. $168-181$

Piris, J.-C. (2012): The future of Europe. Towards a two-speed EU?, Cambridge Prantl, H. (2014): Wo Europa neue Kraft braucht und woher sie kommen kann, in: Hans-Böckler-Stiftung/Deutscher Gewerkschaftsbund/Friedrich-Ebert-Stiftung (Hrsg.): Demokratisierung von Gesellschaft und Arbeitswelt. Impulse für eine soziale Rechtspolitik. Dokumentation des Rechtspolitischen Kongresses, 25./26.3.2015, Berlin

Scharpf, F. W. (1998): Demokratie in der transnationalen Politik, in: Streeck, W. (Hrsg.): Internationale Wirtschaft, nationale Demokratie. Herausforderungen für die Demokratietheorie, Frankfurt a. M., S. 151-174

Scharpf, F. W. (1999): Regieren in Europa. Effektiv und demokratisch?, Frankfurt a. M.

Scharpf, F. W. (2006): The joint-decision trap revisited, in: Journal of Common Market Studies 44 (4), S. 845-864

Scharpf, F. W. (2008a): Negative und positive Integration, in: Höpner, M./Schäfer, A. (Hrsg.): Politische Ökonomie der europäischen Integration, Frankfurt a. M., S. 49-87

Scharpf, F.W. (2008b): „Der einzige Weg ist, dem EuGH nicht zu folgen“, in: Mitbestimmung $07+08 / 2008$, S. $18-23$

Scharpf, F. W. (2009): Legitimität im europäischen Mehrebenensystem, in: Leviathan 37 (2), S. 244-280

Scharpf, F. W. (2010a): The asymmetry of European integration, or why the EU cannot be a 'social market economy', in: Socio-Economic Review 8 (2), S. $211-250$

Scharpf, F. W. (2010b): Negative and positive integration in the political economy of European welfare states, in: Scharpf, F. W. (Hrsg.): Community and autonomy. Institutions, policies and legitimacy in multilevel Europe, Frankfurt a. M., S. 91-126 Scharpf, F. W. (2014): After the crash. A perspective on multilevel European democracy, MPIfG Discussion Paper 14/21, Köln

Scharpf, F. W. (2015): After the crash: a perspective on multilevel European democracy, in: European Law Journal 21 (3), S. 384-405

Schulten, Th./Müller, T. (2013): Ein neuer europäischer Interventionismus?, in: Wirtschaft und Gesellschaft 39 (3), S. 291-321

Schulten, Th. (2015): Konturen einer europäischen Mindestlohnpolitik, in: Alemann, U. v./Heidbreder, E. G./Hummel, H./Dreyer, D./Gödde, A. (Hrsg.): Ein soziales Europa ist möglich. Grundlagen und Handlungsoptionen, Wiesbaden, S. 159-181

Seeliger, M. (2015): Europäischer Mindestlohn als Arbeitnehmerinteresse? Probleme gewerkschaftlicher Positionsbildung, in: Aus Politik und Zeitgeschichte 65 (4-5), S. 36-42

Seikel, D./Absenger, N. (2015): Die Auswirkungen der EuGH-Rechtsprechung auf das Tarifvertragssystem in Deutschland, in: Industrielle Beziehungen 22 (1), S. 51-71 
Streeck, W. (2013): Gekaufte Zeit. Die vertagte Krise des demokratischen Kapitalismus, Berlin

Unger, B. (2013): How to finance a social Europe?, in: Grozelier, A.-M./Hacker, B./ Kowalsky, W./Machnig, J./Meyer, H./Unger, B. (Hrsg.): Roadmap to a social Europe, S. 95-97

Urban, H.-J. (2011): Wirtschaftsdemokratie des 21. Jahrhunderts. Konturen und Realisierungsbedingungen eines gesellschaftlichen Transformationsprojekts, in: Meine, H./Schumann, M./Urban, H.-J. (Hrsg.) (2011): Mehr Wirtschaftsdemokratie wagen!, Hamburg, S. 42-67

Urban, H.-J. (2013): DerTiger und seine Dompteure. Wohlfahrtsstaat und Gewerkschaften im Gegenwartskapitalismus, Hamburg

Ziltener, P. (1999): Strukturwandel der europäischen Integration. Die Europäische Union und die Veränderung von Staatlichkeit, Münster

\section{AUTOR}

DANIEL SEIKEL, Dr., ist Wissenschaftler am Wirtschafts- und Sozialwissenschaftlichen Institut (WSI) in der Hans-Böckler-Stiftung. Arbeitsschwerpunkt: Europäische Politik.

daniel-seikel@boeckler.de 